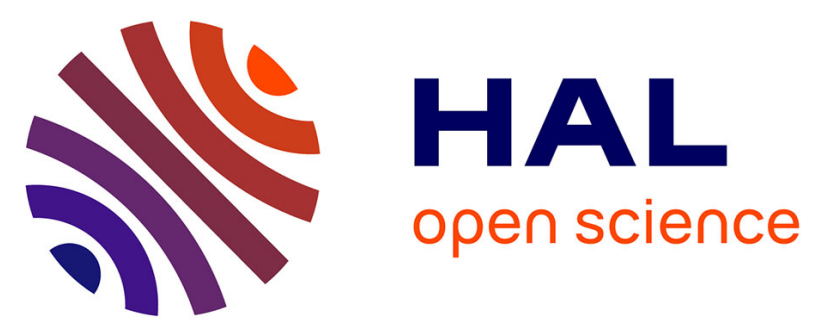

\title{
Miner video tracking and identification using optical camera communications in a wireless multimedia sensor network
}

\author{
Fabian Seguel, Cesar Azurdia-Meza, Nicolas Krommenacker, Patrick \\ Charpentier, Vincent Bombardier, Cristobal Carreño
}

\section{To cite this version:}

Fabian Seguel, Cesar Azurdia-Meza, Nicolas Krommenacker, Patrick Charpentier, Vincent Bombardier, et al.. Miner video tracking and identification using optical camera communications in a wireless multimedia sensor network. 12th International Symposium on Communication Systems, Networks and Digital Signal Processing, CSNDSP 2020, Jul 2020, Porto, Portugal. hal-02983817

\section{HAL Id: hal-02983817 https://hal.science/hal-02983817}

Submitted on 30 Oct 2020

HAL is a multi-disciplinary open access archive for the deposit and dissemination of scientific research documents, whether they are published or not. The documents may come from teaching and research institutions in France or abroad, or from public or private research centers.
L'archive ouverte pluridisciplinaire HAL, est destinée au dépôt et à la diffusion de documents scientifiques de niveau recherche, publiés ou non, émanant des établissements d'enseignement et de recherche français ou étrangers, des laboratoires publics ou privés. 


\section{Miner Video Tracking and Identification Using Optical Camera Communications in a Wireless Multimedia Sensor Network}

\author{
Fabian Seguel \\ Department of Government and Enterprise \\ Universidad de Los Lagos \\ Puerto Montt, Chile \\ fabian.seguelg@usach.cl
}

\author{
Patrick Charpentier \\ Université de Lorraine \\ CNRS, CRAN \\ F-54000 Nancy, France \\ patrick.charpentier@univ-lorraine.fr
}

\author{
Cesar Azurdia-Meza \\ Department of Electrical Engineering \\ Universidad de Chile \\ Santiago, Chile \\ cazurdia@ing.uchile.cl
}

\author{
Vincent Bombardier \\ Université de Lorraine \\ CNRS, CRAN \\ F-54000 Nancy, France \\ vincent.bombardier@univ-lorraine.fr
}

Nicolas Krommenacker

Université de Lorraine

CNRS, CRAN

F-54000 Nancy, France

nicolas.krommenacker@univ-lorraine.fr

\author{
Cristobal Carreño \\ Department of Electrical Engineering \\ Universidad de Santiago de Chile \\ Santiago, Chile \\ cristobal.carreno@usach.cl
}

\begin{abstract}
Following several underground mine disasters, the U.S. government changed its safety policies for underground mines. These new regulations state mandatory wireless communications and tracking of personnel. In this context, wireless multimedia sensor networks have been implemented to provide position information and mine surveillance. The recent development of optical camera communications has enable these networks to provide not only multimedia transmission, but also wireless data communications. In this manuscript, we proposed a novel miner video tracking and identification system using wireless multimedia sensor networks and optical camera communication to overcome the problems of video tracking and identifying personnel inside a challenging scenario such as underground mines.
\end{abstract}

Keywords-Optical camera communications, underground mines, wireless multimedia sensor networks.

\section{INTRODUCTION}

In the recent years, due to the current advance of wireless technologies, new safety standards have been appointed in order to operate underground mines (UM) safely. In June 15, 2006 the congress introduced the PUBLIC LAW 109-236(M) Mine Improvement and New Emergency Response (MINER) [1]. The main goal of this new standard is to provide wireless communications and, position information of personnel inside the mine following an underground accident. The implementation of such systems has encountered various challenges due to the particular conditions of underground mines. Underground mines are considered by several researchers as one of the most difficult indoor scenarios. Underground mining operations involve various hazardous conditions, such as, in-mine vehicular/human accidents, fire and explosions, collapses, toxic gases emanation and floods, among others. In addition to this, workers, machinery and in-mine equipment are exposed to extreme conditions, such as, high levels of humidity (up to 90 $\%$ or above [2]), airborne dust, and extreme heat.

In order to cope with miner communication and tracking challenges, the usage of wireless sensor newtworks (WSNs) for UM monitoring has been widely proposed for researchers [3]-[5]. Wireless multimedia sensor networks (WMSNs) are a kind of WSNs that support multimedia applications, such as the transmission and processing of video, audio, and images [6], [7]. In order to provide tracking services in a WMSNs, video tracking is proposed. Video tracking is the process of locating a single or multiple moving objects over time using a camera. Typically, when people video tracking is implemented, person's body is separated from background image and tracked through the video flux. Nonetheless, to distinguish miner's profile from the mine's background is not possible. This is because mine's background and miners' body are very similar due to insufficient illumination, particles in suspension and dust attached on miners' uniforms.

In order to overcome this problem, miner's cap-lamp video localization has been proposed in recent literature [8], [9] In [8], a 3-D cap-lamp coordinate positioning method using a video collaborative approach is proposed. Although the method provides high localization accuracy and robustness, no extra information is transmitted. In particular, miner identification is not retrieved from video streaming. In [9], a hybrid IMU-video localization method is proposed. A camera base station is used in order to solve the problem of cumulative error in IMU-based position estimation. Miner identification is done by irradiating different light shapes, such as, triangles, rectangles, and circles. Moreover, for uplink data transmission a radio frequency based device is used.

In this article a miner video tracking and identification method using optical camera communications (OCC) in a 
WMSNs is proposed. Our method uses rolling shutter (RS) based OCC to transmit miner's identification. Once the miner is identified and data is demodulated, a video-tracking method is performed in order to localize the mobile user within the video frame. Our method overcome the following problems encountered when tracking and identifying miners inside and underground tunnel:

1) Detect miner location inside the video frame with insufficient light conditions

2) Identify the miner despite the insufficient light conditions by re-using helmet mounted lamps and OCC

3) Track the miner movements inside the mine

4) Provide a large communications coverage range in underground tunnels without increasing the required WMSN infrastructure

This article is organized as follows. In Section II, the proposed system to provide WMNSNs and OCC in a underground mine environment is delivered. Transmitter and receiver hardware as well as the testing scenario are described. In Section III, the identification and localization methods are detailed. Then, in Section IV the main results of our study are discussed. Finally, in Section V the main conclusions of this work are delivered.

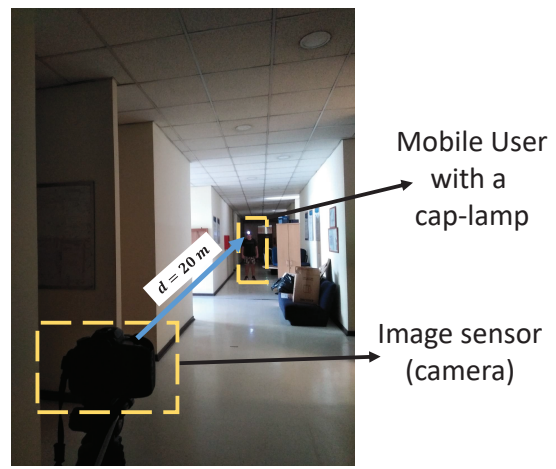

(a)

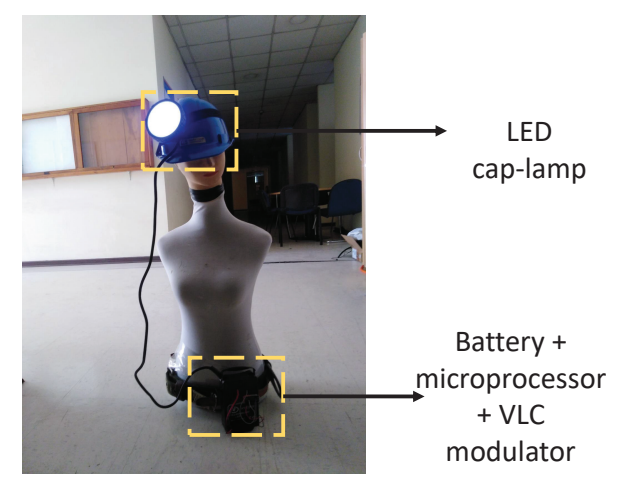

(b)

Fig. 1. Proposed system diagram for remote positioning in WMSNs (a) overall scenario and (b) mobile user transmitter device.

\section{SYSTEM DESCRIPTION}

In order to test our method, we deploy a small WMSN using a single camera to provide miner tracking and identification into a twenty meters large indoor hall as shown in Figure 1 (a). The mobile user (miner) is equipped with a cap-lamp connected to an in-belt battery coupled with an OCC modulator as depicted in Figure 1 (b). The main transmitter and receiver components are detailed in the following subsections.

\section{A. Camera receiver}

On the basis of the pixel exposure, cameras can be classified into two categories, rolling shutter (RS) and global shutter (GS). GS employs mostly charge coupled device (CCD) image sensors. In global shutter cameras all pixels are exposed simultaneously in a frame. RS cameras employ complementary metal-oxide-semiconductor (CMOS) image sensors. This type of cameras scan pixels sequentially [10]. Rolling shutter (RS) OCC based uses PWM banding effect while capturing LED light sources. RS CMOS cameras are used constructively in the case of OOK signal decoding. When doing this, the camera OOk modulated LED outputs are bright and dark bands. Bright bands represent the bit "1", whilst dark bands represent the bit "0". Recent advances of rolling-shutter based OCC have enabled large distance wireless communications of up to 400 $m$ by defocusing the Camera Lens [11]-[13]. At the receiver side, a Canon EOS 60D camera is used to record the scene. The video flux is transmitted to a laptop using the Wifi network. The received video is processed using MATLAB.

\section{B. Cap lamp transmitter}

The transmitter must rapidly switch the LEDs. In addition to this, the on-interval and off-interval must be distributed in such a way that the human's eyes cannot perceive the LEDs' flickering. The LED pulsating frequency is kept lower than the rolling shutter's scanning frequency, but higher than the camera frame rates [10]. The VLC transmitter is composed by an Arduino Nano which stores the miner ID and command the OOK signal. The cap-lamp is coupled with a dual MOSFET trigger switch. The data transmitted by the in-helmet transmitter is shown in Figure 2.

\begin{tabular}{|c|c|}
\hline FH & $\begin{array}{c}\text { Manchester coded } \\
\text { Miner ID } \\
\text { (11110) }\end{array}$ \\
\hline
\end{tabular}

Fig. 2. VLC packet transmitted by the cap-lamp.

The transmitted packet is composed by the frame header and a 2 bits miner ID which is converted to 4 symbols by using Manchester coding. The frame header is composed by a 4-bit logic high symbol that never occurs in the normal Manchester coding data followed by an "OFF" signal for clock synchronization purpose.

\section{MinER LOCALIZATION AND IDENTIFICATION}

In order to identify and localize miners inside the underground mine tunnel, cap lamp pixels must be separated from background environment. Once the region of interest (ROI) is extracted in the video frame, identification packet is demodulated from the video stream by using the ROI pixels as 
shown in Figure 3. After the miner ID information is retrieved, it is used to display the miner identification at the monitoring central station overground.

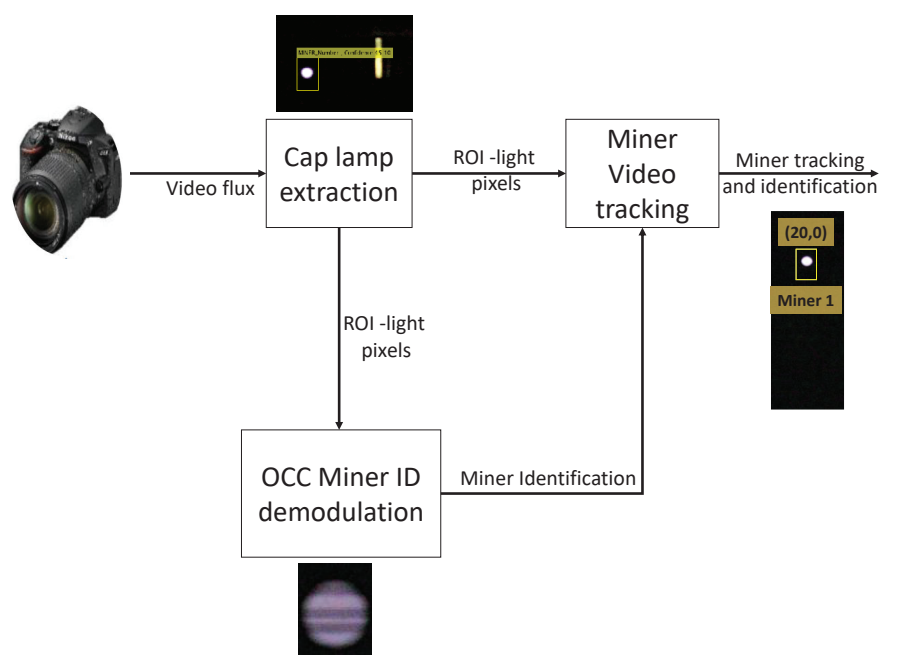

Fig. 3. Proposed method for miner identification and localization.

A detailed explanation of each step of the process is delivered next.

\section{A. Cap-lamp extraction}

In order to determine the light ROI within the frame, aggregate channel features (ACF) method described in [14] is used. A 21 seconds length video is divided in two sets. The training set correspond to the first 5 seconds of the video. Once the method has extracted the main features to perform the cap-lamp extraction, the remaining 16 seconds of the video are used for method validation and performance evaluation.

\section{B. Miner identification}

Miner identification information is retrieved directly from the video. Once the ROI is determined, pixels of interest are processed. Miner ID is retrieved from ROI by integrating the light intensity of each pixel line [15]. In order to increase the communication distance between the RS camera and cap-lamp, blurred image of a light source (LS) proposed in [12] is used. This method allows us to perform RS-OCC with an extended communication range. In Figure 4 (b) the defocusing effect of a light source can be seen. The distance between the camera and the light source is $5 \mathrm{~m}$ in both cases. Size of blurred light source is larger than unblurred light shown in Figure 4 (a). Due to this, line by line demodulation and data recovery is easier to perform.

\section{Miner tracking}

In order to track the movement of the miner, ROI is used to perform the tracking of the cap lamp within a specific video frame. A bounding box is obtained as a result of the cap-lamp extraction. The immediate position of the light within the video frame can be assumed as the center of this bounding box as follows:

$$
(\hat{x}, \hat{y})=\left(\frac{x_{\min }+x_{\max }}{2}, \frac{y_{\min }+y_{\max }}{2}\right),
$$

where $\hat{x}$ and $\hat{y}$ is the estimated position of the cap lamp within the frame and $x_{\min }, x_{\max }, y_{\min }$ and $y_{\max }$ are the bounding box vertices.

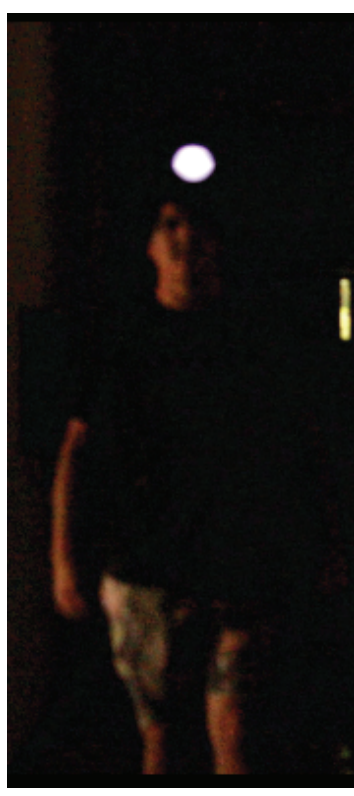

(a)

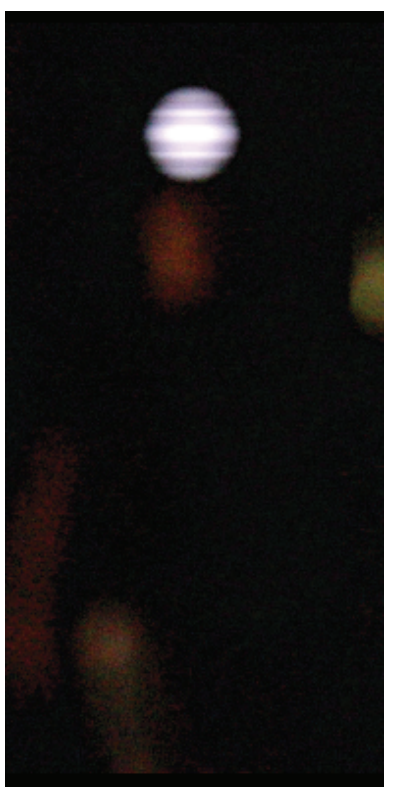

(b)
Fig. 4. Comparison of light size within the frame by using (a) unblurred light source and, (b) blurred light source.

\section{RESUlts}

Figure 1 illustrates the experimental setup for evaluation of the proposed system. In order to simulate UM conditions of insufficient background illumination, videos were taken in dark hours using the hall section without windows and lights off. This proof of concept tried to simulate as close as possible the conditions inside the mine. Nonetheless, airborne dust has not been considered in this essay. In addition to this, at this early stage of the study some other aspects, such as, multiple access and shadowing have not yet been studied. Nonetheless, since spatial multiplexing is an inherent capability in OCC systems, it will be used in further developments.

In Table I camera and set up parameters used for experimental demonstration are delivered.

TABLE I

SYSTEM PARAMETERS

\begin{tabular}{|c|c|c|c|}
\hline Parameter & Value & Parameter & Value \\
\hline Shutter speed & $1 / 4000$ & ISO & 1600 \\
\hline Camera lens & $35 \mathrm{~mm} \mathrm{~F} / 2.0$ & Frame rate & $50 \mathrm{fps}$ \\
\hline Data rate & $0.5 \mathrm{Kbps}$ & Max. distance & $20 \mathrm{~m}$ \\
\hline Lamp diameter & $0.1 \mathrm{~m}$ & LED power & $3 \mathrm{~W}$ \\
\hline
\end{tabular}

In Figure 5 the performance of the cap-lamp detector in terms of precision, recall, and miss rate is shown. An overlapping threshold of $T H=0.5$ is used in order to determine 
whether the detection is correct or not is used. Precision, recall, and, miss rate are defined as follows:

$$
\begin{aligned}
\text { Precision } & =\frac{T P}{T P+F P}, \\
\text { Recall } & =\frac{T P}{T P+F N}, \\
\text { Miss rate } & =\frac{F N}{T P+F N},
\end{aligned}
$$

where TP, FP, and FN are the true positives, false positive and false negative cap lamp extractions. Given the above mentioned results, we can ensure ROI determination with a, average precision of 0.8 .

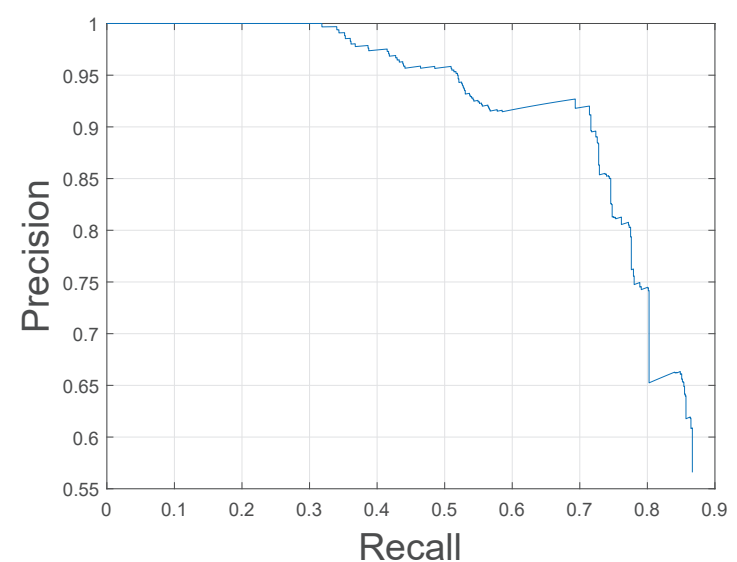

(a)

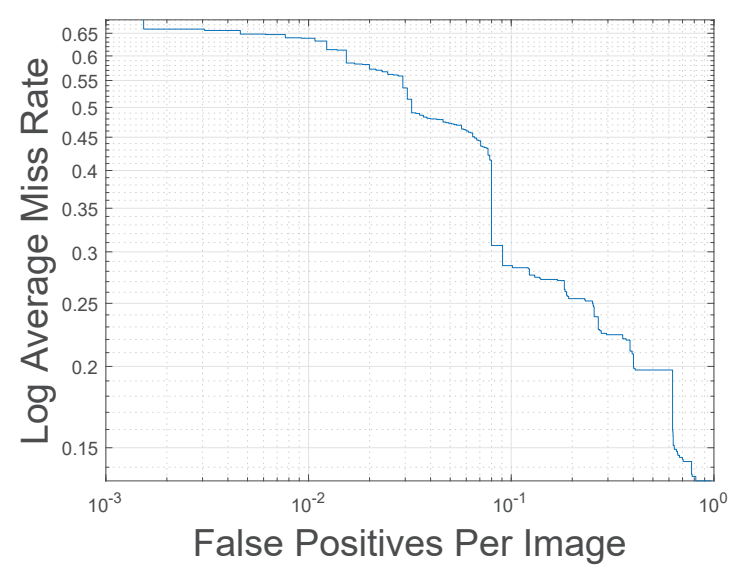

(b)

Fig. 5. Performance of cap-lamp extraction (a) Average precision and (b) log average miss rate

Another important metric is the miner identification rate which is given by

$$
\text { Miner identification rate }=\frac{T D}{N_{\text {users }}},
$$

where $T D$ is the number of correct ID detection and $N_{\text {users }}$ is the total number of users within the environment. Depending on the distance between transmitter and receiver, performance of miner identification can decrease due to the bit error rate (BER). Fortunately, due to the insufficient illumination inside the tunnel, background noise is very low in this scenario and miner ID is easily obtained from video flux as shown in Figure 6. In our proposal a single user is considered. Due to this, we

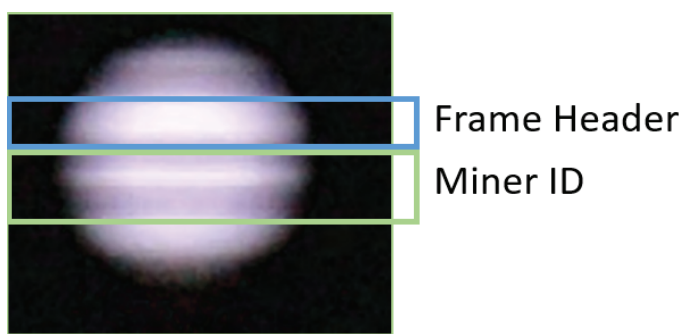

Fig. 6. ROI containing miner identification

have achieved $100 \%$ percent of miner identification rate even for a Light-to-camera distance of $20 \mathrm{~m}$.

In Figure 7, cap-lamp tracking over a video of $20 \mathrm{~s}$ duration is displayed. Miner walks straight towards the video camera from a maximum distance of 20 meters. As it can be seen, due to the frontal orientation and the low height of the image sensor, light position height becomes higher as the mobile user approaches to the camera. Vertical position change is larger than horizontal movement. Small periodic horizontal movements caused by footsteps are present in the tracking.

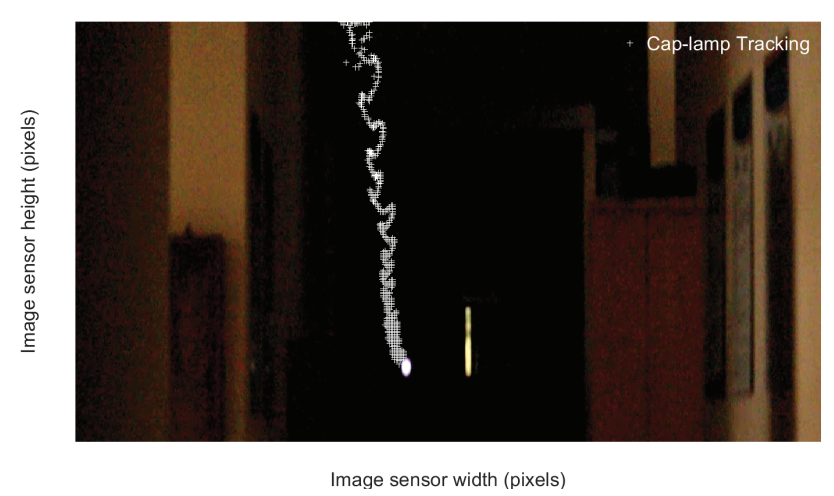

Fig. 7. Cap lamp tracking over a 20 meters straight walking towards the image sensor: starting frame

The proposed method is capable to track the mobile RSOCC transmitter.

\section{CONCLUSIONS}

In this manuscript the proof of concept of a novel miner video tracking and identification system is proposed. The method uses a WMSN and RS-OCC to provide the before mention services. By using the ACF method, we were capable to extract the cap-lamp from the background scene with an $80 \%$ precision. Moreover, wireless data transmission in a distance of up to $20 \mathrm{~m}$ is performed by defocusing the video frame. In the proposed scenario, the miner was identified with a $100 \%$ of accuracy even for the maximum testing distance. In addition to this, the method is capable to track light movement 
within the video flux showing the potential of our system to provide miner video tracking in the proposed WMSN. As future work, more precise light extraction methods will be developed. Moreover, real world miner's coordinates inside the tunnel will be obtained. Few real scenario conditions such as multiplexing, shadowing and the effect of user dynamics in the data transmission will be measured. Finally, the proposed method will be evaluated in a real underground mine tunnel.

\section{ACKNOWLEDGMENT}

The authors acknowledge the financial support of Beca Doctorado Nacional 2016 CONICYT (PFCHA) 21161397, STIC Am Sud 19-STIC-08, and Fondef Proyecto IT17M10012.

\section{REFERENCES}

[1] 109th Congress, "Mine Improvement and New Emergency Response," 2006

[2] S. Yarkan, S. Guzelgoz, H. Arslan, and R. Murphy, "Underground Mine Communications: A Survey," IEEE Communications Surveys \& Tutorials, vol. 11, no. 3, pp. 125-142, 2009.

[3] M. Li and Y. Liu, "Underground coal mine monitoring with wireless sensor networks," ACM Transactions on Sensor Networks, vol. 5, pp. 129, mar 2009.

[4] Z. Liu, C. Li, D. Wu, W. Dai, S. Geng, and Q. Ding, "A wireless sensor network based personnel positioning scheme in coal mines with blind areas," Sensors (Switzerland), vol. 10, no. 11, pp. 9891-9918, 2010.

[5] P. Marko and S. Pirjo, Industrial Wireless Sensor Networks. Elsevier, 2016.

[6] I. F. Akyildiz, T. Melodia, and K. R. Chowdury, "Wireless multimedia sensor networks: A survey," dec 2007.
[7] I. F. Akyildiz, T. Melodia, and K. R. Chowdhury, "A survey on wireless multimedia sensor networks," Computer Networks, vol. 51, pp. 921-960, mar 2007.

[8] K. You, W. Yang, and R. Han, "The video collaborative localization of a miner's lamp based on wireless multimedia sensor networks for underground coal mines," Sensors (Switzerland), vol. 15, no. 10, pp. 25103-25122, 2015.

[9] Q. Niu, X. Yang, and Y. Yin, "IPL: Image-Assisted Person Localization for Underground Coal Mines," Sensors, vol. 18, p. 3679, oct 2018.

[10] N. T. Le, M. S. Ifthekhar, Y. M. Jang, and N. Saha, "Survey on optical camera communications: challenges and opportunities," IET Optoelectronics, vol. 9, no. 5, pp. 172-183, 2015.

[11] E. Eso, S. Teli, N. Bani Hassan, S. Vitek, Z. Ghassemlooy, and S. Zvanovec, "400 m rolling-shutter-based optical camera communications link," Optics Letters, vol. 45, p. 1059, mar 2020.

[12] N. B. Hassan, Y. Huang, Z. Shou, Z. Ghassemlooy, A. Sturniolo, S. Zvanovec, P. Luo, and H. Le-Minh, "Impact of Camera Lens Aperture and the Light Source Size on Optical Camera Communications," in 2018 11th International Symposium on Communication Systems, Networks and Digital Signal Processing, CSNDSP 2018, Institute of Electrical and Electronics Engineers Inc., sep 2018.

[13] N. Hassan, Y. Huang, Z. Shou, Z. Ghassemlooy, S. Zvanovec, M. Zhang, X. Tang, and N. B. Hassan, "Impact of Defocusing of the Camera Lens on the Image in Optical Camera Communications. West Asian Colloquium on Optical Wireless Communications," tech. rep., apr 2018.

[14] P. Dollar, R. Appel, S. Belongie, and P. Perona, "Fast feature pyramids for object detection," IEEE Transactions on Pattern Analysis and Machine Intelligence, vol. 36, pp. 1532-1545, aug 2014.

[15] A. Sturniolo, G. Cossu, E. Ciaramella, N. B. Hassan, Z. Shou, Y. Huang, and Z. Ghassemlooy, "ROI Assisted Digital Signal Processing for Rolling Shutter Optical Camera Communications," in 2018 11th International Symposium on Communication Systems, Networks and Digital Signal Processing, CSNDSP 2018, Institute of Electrical and Electronics Engineers Inc., sep 2018. 